Nouvelles perspectives en sciences sociales

Revue internationale de systémique complexe et d'études relationnelles

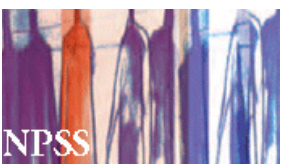

\title{
Aux racines de la « bonne sociologie »
}

\section{Raymond Boudon}

Volume 8, numéro 1, novembre 2012

URI : https://id.erudit.org/iderudit/1013920ar

DOI : https://doi.org/10.7202/1013920ar

Aller au sommaire du numéro

Éditeur(s)

Prise de parole

ISSN

1712-8307 (imprimé)

1918-7475 (numérique)

Découvrir la revue

Citer cet article

Boudon, R. (2012). Aux racines de la « bonne sociologie ». Nouvelles perspectives en sciences sociales, 8(1), 119-160. https://doi.org/10.7202/1013920ar
Résumé de l'article

Les travaux sociologiques considérés comme majeurs suivent trois règles communes à toutes les disciplines scientifiques. Elles sont à la racine du paradigme sociologique qui s'installe aujourd'hui sous l'étiquette de la sociologie analytique. Ces règles sont cruciales s'agissant de tous les sujets d'intérêt pour le sociologue. On le suggérera à l'exemple de l'explication que proposent des travaux sociologiques classiques et modernes des croyances collectives représentationnelles et normatives, de leur changement dans le temps et de leurs différences d'une société à l'autre. 


\section{Aux racines de la "bonne sociologie $\|^{1}$}

\section{RAYMONd Boudon}

Académie des sciences morales et politiques

$L_{\text {es Trois cultures de Wolf Lepenies }}^{2}$, un livre de réflexion sur sociologiques dans les dernières années du siècle passé. Il a été rapidement traduit dans les principales langues européennes. Il défend deux thèses : que la sociologie n'est ni une science ni un art, mais une "tierce culture " et que les grands sociologues classiques se sont bercés d'illusion en croyant que la sociologie pouvait être une science comme les autres. Le livre tirait peut-être son inspiration de l'état de fait de la sociologie à l'époque où il a été écrit, dans les années 1980. Car, en ce temps, deux mouvances intellectuelles obéissant à deux visions diamétralement opposées dominent la sociologie, le structuralisme et le constructivisme. La première est pétrie de scientisme, la seconde de relativisme. Aujourd'hui, un cycle nouveau parait s'ouvrir avec le déploiement de ce qu'on appelle couramment désormais la sociologie analytique ${ }^{3}$. Elle reprend le paradigme auquel Weber

1 Version française d'une communication inédite donnée en anglais au Centre culturel norvégien de Paris le 5 janvier 2012 dans le cadre d'un cycle d'enseignement doctoral de l'Université d'Oslo.

2 Wolf Lepenies, Die drei Kulturen, Munich, Hanser, 1985. En français : Les trois cultures, Paris, Maison des sciences de l'homme, 1991.

3 Voir Pierre Demeulenaere, "Mécanismes, causalité, régularités de comportements et normes sociales ", dans Gérald Bronner et Romy Sauvayre (dir.), Le 
associa la notion de "sociologie compréhensive " ou Merton celle de " middle range theory ". En allemand, on parle plutôt se " sociologie explicative" (Erklärende Soziologie).

Un commentaire de la quatrième de couverture d'un ouvrage collectif publié sous la direction de Pierre Demeulenaere, Analytical Sociology and Social Mechanisms, déclare que la sociologie analytique, c'est tout simplement la "bonne sociologie » (good sociology), suggérant que la sociologie analytique représente essentiellement une réaction contre le degré croissant de diversité qui caractérise la sociologie depuis les années $1960^{4}$. Je pense que la sociologie analytique est effectivement le symptôme de la fin du cycle " ni science, ni art » et qu'elle retrouve les ambitions des sociologues classiques : faire de la sociologie une science comme les autres.

Je tenterai dans ce qui suit de développer deux thèses : d'abord que bien des travaux sociologiques considérés comme d'authentiques contributions scientifiques suivent trois règles communes à toutes les sciences, ensuite, que ces règles sont situées à la racine de la sociologie analytique. Comme je l'ai montré ailleurs ${ }^{5}$, une explication scientifique d'un phénomène $\mathrm{X}$ quelconque peut se définir comme un ensemble $\mathrm{E}$ de propositions répondant à trois critères : 1) évoquant toutes des lois ou des phénomènes avérés 2) d'où l'on déduit $X$ et 3) tel qu'on ne connaît pas dans l'état actuel du savoir d'ensemble alternatif E' rendant aussi et mieux compte de X. Je me concentrerai sur l'importance de ces règles pour l'explication des croyances représentationnelles et normatives, étant entendu que j'aurais pu emprunter mes exemples à d'autres chapitres de la sociologie. Elles permettent de com-

Naturalisme dans les sciences sociales, Paris, Hermann, 2011, p. 167-182; Peter Hedström, Dissecting the Social: on the Principles of Analytical Sociology, Cambridge, Cambridge University Press, 2005; Gianluca Manzo, «Analytical Sociology and Its Critic », European Journal of Sociology, vol. 51, n 1, 2010, p.129-170.

4 Pierre Demeulenaere, (dir.), Analytical Sociology and Social Mechanisms, Cambridge, Cambridge University Press, 2010.

5 Raymond Boudon, La Sociologie comme science, Paris, La Découverte, coll. "Repères ", 2011; Raymond Boudon, Croire et savoir : penser le politique, le moral et le religieux, Paris, Presses universitaires de France, 2012. 
prendre pourquoi ces croyances sont différentes d'une société et d'une époque à l'autre et aussi pourquoi des effets de contexte limitent l'influence des processus d'homogénéisation à l'œuvre dans les sociétés contemporaines.

\section{Le singularisme méthodologique}

Émile Durkheim, celui que tous les manuels considèrent, avec Max Weber, comme le père de la sociologie, se pose dans son étude sur Le Suicide une série de questions portant toutes sur des phénomènes singuliers : pourquoi les taux de suicide des femmes sont-ils régulièrement plus bas que ceux des hommes selon les statistiques disponibles sur le sujet à la fin du XIX ${ }^{\mathrm{e}}$ siècle? Pourquoi les taux de suicide des protestants sont-ils plus élevés que ceux des catholiques? Pourquoi ceux des célibataires sont-ils plus élevés que ceux des personnes vivant en famille? Durkheim s'efforce ensuite de synthétiser les explications qu'il propose de cet ensemble de faits singuliers dans le cadre théorique plus large de ses trois types de suicide, qui sont en fait quatre, comme les trois mousquetaires.

Max Weber épouse la même démarche. Ainsi, il se demande dans son Judaïsme antique pourquoi les Pharisiens croient à l'immortalité de l'âme et pourquoi les Sadducéens n'y croient guère. Ailleurs, il s'interroge sur les raisons d'être de l'exception religieuse américaine : pourquoi les Américains restent-ils beaucoup plus religieux que les Anglais, les Français ou les Allemands? L'accumulation des explications qu'il propose de phénomènes singuliers comme ceux-là lui permet de renouveler l'explication des phénomènes religieux.

C'est le grand économiste autrichien Ludwig von Mises qui a forgé la notion de singularisme méthodologique pour identifier la démarche qui se donne pour objet l'explication de phénomènes singuliers, et la distinguer de celle qui cherche à embrasser de vastes objets d'un regard global ${ }^{6}$. Pour von Mises, le singularisme

$6 \quad$ Ouvrage majeur de Ludwig von Mises, L'Action humaine, sous-titré Traité d'économie (Paris, Presses universitaires de France, 1985), a été publié en 1949 en anglais par les presses de l'Université de Yale (New Haven (CT) sous le titre Human Action : A Treatise on Economics. 
méthodologique est une condition de toute explication scientifique. La perspective qu'on peut qualifier par symétrie de holisme méthodologique peut conduire à des interprétations intéressantes, mais non à des explications au sens des trois critères précédemment évoqués. Ainsi, une séquence complexe d'événements comme la Révolution française de 1789 ne peut être expliquée en ce sens, ne fût-ce que parce qu'elle correspond à un phénomène par essence mal défini. On ne peut notamment déterminer avec certitude son point final. Pour certains historiens, elle s'est achevée avec la prise de pouvoir par Bonaparte en 1800. Selon François Furet, elle s'est terminée en 1870, avec la Commune de Paris. En revanche, ce sujet peut donner lieu à interprétation. Georg Simmel a développé dans un grand livre l'idée que certains sujets peuvent donner lieu à interprétation, d'autres à explication' Ainsi, on ne conçoit pas qu'aucune biographie d'un grand acteur historique puisse être définitive, toute biographie reposant sur une sélection et une pondération différentielle d'un nombre indéfini de faits, lesquelles sont obligatoirement affectées par la sensibilité de l'historien et de son temps. On ne conçoit pas non plus une interprétation définitive de la Révolution qui interdirait tout nouveau livre sur le sujet. En revanche, on peut expliquer pourquoi les taux de suicide de telle catégorie sociale sont dans certaines conjonctures supérieurs à ceux de telle autre ou pourquoi les Américains apparaissent comme beaucoup plus religieux que les Européens. Bien des explications de phénomènes singuliers de ce type n'ont jamais été remises en question, comme le suggèrent les exemples développés ci-après.

Si j'insiste d'entrée de jeu sur la notion du singularisme méthodologique, c'est qu'elle s'applique, non seulement à la sociologie, mais à toutes les sciences sociales. Toutes ont donné naissance à des traditions de pensée obéissant au principe du singularisme méthodologique ou à son contraire.

Que la notion du singularisme méthodologique ait été créée par un économiste autrichien ne doit rien au hasard. À la fin du XIX

Georg Simmel, Die Probleme der Geschichtsphilosophie, Munich, Mohr, 1892. 
et au début du $\mathrm{XX}^{\mathrm{e}}$ siècle, les économistes allemands et autrichiens donnent en effet l'impression d'appartenir à deux mondes intellectuels différents. Du côté des Allemands, domine le courant de pensée dit de l'école historique, qu'illustre notamment Gustav Schmoller. En simplifiant, il affirme que l'économie a pour objectif de saisir l'évolution des institutions économiques. Les économistes allemands regardent alors de haut les jeunes économistes autrichiens qui, comme les marginalistes, développent des modèles visant à expliquer des phénomènes économiques singuliers. Leur opposition a donné lieu à une querelle de méthode restée célèbre dans l'histoire des idées économiques.

L'histoire mouvementée de la célèbre École sociologique de Francfort illustre, elle aussi, ce même conflit de méthode. Je me contente d'en extraire une anecdote ${ }^{8}$. Fuyant la montée du national-socialisme en Allemagne, plusieurs représentants de l'École de Francfort sont accueillis aux États-Unis dans les années 1930. Max Horkheimer, la figure centrale de l'École, se propose d'y développer un projet situé dans la filiation de Marx et visant à développer une Théorie de la société dont il insiste pour la présenter avec un grand $\mathrm{T}$, ce qui est banal en allemand, mais insolite en anglais. Ayant quelque peine à comprendre son projet, ses interlocuteurs américains renâclent à l'encourager. Dans un premier temps, un sociologue d'origine autrichienne encore peu connu et qui allait devenir l'un des sociologues les plus importants du XX $\mathrm{XX}^{\mathrm{e}}$ siècle, Paul Lazarsfeld, lui apporte son appui. Mais dès que Horkheimer et Lazarsfeld parvinrent à s'ancrer dans le monde universitaire américain, ils se regardèrent en chiens de faïence. La raison principale en est que, à l'instar des économistes allemands et autrichiens, ils s'étaient rangés des deux côtés de la fracture du singularisme.

$\overline{8}$ Rolf Wiggershaus, The Frankfurt School, Norristown (PA), 1995. En français, L'École de Francfort, Paris, Presses universitaires de France, 1993, présentation et traduction de Lilyane Deroche-Gurcel. 


\section{Les fondateurs de la sociologie adoptent le singularisme méthodologique}

En épousant le principe du singularisme méthodologique, les pères fondateurs de la sociologie visaient à expliquer des phénomènes sociaux énigmatiques à l'aide des procédures qui sont celles de toutes les sciences. Ainsi, dans son travail courant, le biologiste se penche sur les causes de phénomènes singuliers, comme l'action de tel ou tel virus. Il ne se désintéresse pas de la question de l'essence et de l'origine de la vie, mais il la repousse à l'horizon de sa recherche.

Max Weber obéit aux mêmes principes que le biologiste lorsqu'il explique pourquoi les Pharisiens croyaient à l'immortalité de l'âme, alors que les Sadducéens n'y croyaient guère?. Cela résulte, explique-t-il, de ce que les Pharisiens étaient en majorité des commerçants. L'équité des échanges représentant une valeur cruciale pour eux, ils étaient heureux d'apprendre que l'âme est immortelle, puisque cela leur permettait d'espérer que les mérites et les démérites qui n'avaient pas reçu leur juste sanction ici-bas feraient l'objet d'une révision dans l'au-delà. L'idée de l'immortalité de l'âme leur faisait miroiter sur le mode symbolique l'assurance que leur souci d'équité serait satisfait. Les Sadducéens, eux, constituaient le vivier d'où étaient puisées les élites politiques juives. Ils n'avaient donc pas les mêmes raisons d'adhérer à une idée venue d'ailleurs, de l'Inde sans doute, et qui leur paraissait étrange.

C'est de la même manière que Tocqueville ${ }^{10}$, devançant ici Max Weber, avait proposé d'expliquer la croyance hindouiste à la métempsycose. Selon lui, la suite des réincarnations d'un même être traduit de façon symbolique le souci du croyant que justice lui soit rendue en une sorte de jugement dernier, au terme du cycle de ses réincarnations.

9 Max Weber, Gesammelte Aufsätze zur Religionssoziologie, Munich, Mohr, 1986 [1920].

10 Alexis de Tocqueville, La Démocratie en Amérique II, Paris, Gallimard, 1986 [1840], p. 527. 
Autre exemple de singularisme méthodologique : l'explication proposée par Durkheim des raisons pour lesquelles la notion de miracle est facilement évoquée pendant des siècles, puis déconsidérée. C'est que la notion de miracle, explique-t-il, ne pouvait être discréditée qu'à partir du moment où le développement des sciences eût solidement installé la notion de loi de la nature et imposé l'idée du déterminisme. Auparavant, rien ne s'opposait à ce que bien des événements soient perçus comme le produit de pratiques que la modernité a qualifiées depuis de magiques. Avant le désenchantement $d u$ monde, il était normal que la notion de miracle aille de soi, notamment pour ceux qui partageaient les croyances chrétiennes. Après, il était compréhensible qu’elle soit discréditée.

J'ai voulu insister pour commencer sur le contraste entre la sociologie visant à l'explication scientifique de faits singuliers énigmatiques et la sociologie cherchant à saisir les sociétés comme des totalités, parce que ce contraste est permanent.

Ainsi, le sociologue allemand Ulrich Beck ${ }^{11}$ doit sa popularité actuelle à ce qu'il a créé l'expression société $d u$ risque (Risikogesellschaft), par laquelle il tente de saisir l'essence des sociétés contemporaines. Le sociologue anglo-polonais Zygmunt Baumann doit la sienne à ce qu'il y voit des sociétés liquides (liquid societies), sans points fixes, où toutes les valeurs et toutes les institutions auraient perdu leur solidité d'antan ${ }^{12}$. À la fin du $\mathrm{XIX}^{\mathrm{e}}$ siècle, Gustave Le Bon avait atteint à une audience que Weber et Durkheim n'ont jamais connue, parce qu'il décrivit les sociétés de son temps comme caractérisées par un phénomène inédit selon lui : le règne des foules ${ }^{13}$.

Il était indispensable d'évoquer cette sociologie de caractère holiste, car elle est depuis toujours la plus visible. Mais je voudrais maintenant me tourner vers la sociologie à ambition scientifique, celle qui voit la sociologie comme ayant vocation à suivre les procédures et les principes auxquels obéissent toutes les sciences.

$11 \quad$ Ulrich Beck, Risk Society: Towards a New Modernity, London, Sage, 1992.

12 Zygmunt Baumann, Does Ethics Have a Chance in a Word of Consumers?, Cambridge (MA), Harvard University Press, 2008.

13 Gustave Le Bon, Psychologie des foules, Paris, Alcan, 1895. 
Le singularisme méthodologique représente l'un de ces principes, mais il en est d'autres.

\section{L'individualisme méthodologique}

Ainsi, le singularisme méthodologique est la première règle commune à toutes les explications scientifiques dans les sciences sociales. Max Weber et Joseph Schumpeter, qui a travaillé un temps pour le compte de Max Weber, ont identifié une seconde règle et l'ont désignée par l'expression individualisme méthodologique. La notion a ensuite été popularisée notamment par Karl Popper et Friedrich Hayek. Max Weber voyait l'individualisme méthodologique comme le principe de base de ce qu'il appelait la sociologie compréhensive : "la sociologie compréhensive au sens où je prends cette expression considère l'individu isolé et son action comme son unité ultime, comme son atome" (die verstehende Soziologie, in unserem Sinne, behandelt das Einzelindividuum und sein Handeln als unterste Einheit, als ihr 'Atom' ${ }^{14}$. On ne saurait être plus clair : l'individualisme méthodologique est le principe de base de la sociologie compréhensive parce que les actions individuelles sont les seules causes possibles des phénomènes sociaux. Malheureusement, les manuels interprètent souvent à tort l'individualisme méthodologique comme impliquant la méconnaissance du fait que les individus sont plongés dans des institutions et, plus généralement, appartiennent à un contexte social et ont une histoire. Les promoteurs de la sociologie analytique ont peut-être décidé de recourir à une expression inédite, notamment pour contourner ce contresens courant.

Il faut noter que la notion de sociologie compréhensive est souvent elle-même mal comprise. C'est pourquoi Weber précise dans le texte cité qu'il prend la notion en un sens à lui. En effet, bien des sociologues de son temps donnaient souvent à cette expression un sens holiste plutôt qu'individualiste. Aujourd'hui encore, certains manuels décrivent la sociologie compréhensive comme celle qui tente de décrire les traits caractéristiques de

14 Max Weber, Gesammelte Aufsätze zur Wissenschaftslehre, Tübingen, Mohr, 1922. En français : Essai sur la théorie de la science, Paris, Plon, 1965, p. 415. 
certaines sociétés ou de certaines époques, comme Dilthey ou Burckhardt hier ou Ulrich Beck et Zygmunt Baumann aujourd'hui. Une petite histoire des contresens affectant les notions de compréhension (Verstehen) et d'individualisme méthodologique mériterait d'être écrite.

Contre ces confusions, Weber a clairement défendu l'idée qu'expliquer des phénomènes singuliers est l'objectif principal de la sociologie et que, comme les phénomènes sociaux ne peuvent avoir d'autre cause que des actions humaines individuelles, les expliquer suppose que le sociologue soit en état de déterminer les causes desdites actions. C'est ce qu'il fait lorsqu'il explique par exemple pourquoi les Pharisiens croyaient à l'immortalité de l'âme, alors que les Sadducéens n'y croyaient pas. La cause de cette différence macro réside dans les raisons qui faisaient qu'un Pharisien idéal-typique avait davantage de chances d'être séduit par l'idée qu'un Sadducéen idéal-typique. Cette analyse ne suppose pas que les individus concrets aient clairement perçu les raisons qui les guidaient. Car les raisons qui animent l'acteur social sont en effet plus souvent métaconscientes que conscientes.

Contre les vues défendues dans nombre de manuels, Durkheim a, lui aussi, implicitement adopté le principe de l'individualisme méthodologique, en dépit du caractère confus de ses considérations sur le lien micro-macro qui donnent l'impression qu'il le rejette $^{15}$. Ainsi, dans son Suicide, il explique le fait macro que les protestants se suicident plus fréquemment que les catholiques à l'aide de l'hypothèse que le catholique idéal-typique se laisse guider par les instructions du magistère, tandis que le protestant doit résoudre ses problèmes existentiels en déchiffrant par luimême les recommandations des textes sacrés. D'où le fait que le

$\overline{15}$ Massimo Borlandi, "Durkheim et la psychologie », dans Raymond Boudon (dir.), Durkheim fut-il durkheimien?, Actes du colloque organisé les 4 et 5 novembre 2008 par l'Académie des sciences morales et politiques, Paris, Colin, coll. "Recherches ", 2011, p. 55-80; Mohamed Cherkaoui, " La Complexité du social et la méthode sociologique selon Durkheim ", dans Raymond Boudon (dir.), Durkheim fut-il durkheimien?, Actes du colloque organisé les 4 et 5 novembre 2008 par l'Académie des sciences morales et politiques, Paris, Colin, coll. « Recherches », 2011, p. 81-98. 
second a plus de chances en cas d'échec de recourir à la «solution $»$ du suicide.

Ou, pour prendre un exemple tiré du dernier livre de Durkheim sur Les Formes élémentaires de la vie religieuse, il explique que la notion d'âme soit universelle et, par contraste avec d'autres notions religieuses, hautement durable, en montrant qu'elle a une signification symbolique qui s'impose facilement à tout un chacun. Dans toute société, déclare-t-il, chaque individu a le sentiment que certaines conduites sont légitimes ou non, bonnes ou non. Dans notre vocabulaire moderne d'inspiration nietzschéenne, tout individu a un sens des valeurs. De plus, il a le sentiment que les valeurs auxquelles il est attaché viennent de l'extérieur. En conséquence, il ne peut éviter d'éprouver un sentiment de dualité de son moi. Selon Durkheim, la notion d'âme doit être interprétée comme une expression symbolique de cette dualité. Des sociologues contemporains des religions, comme Thomas Luckman ${ }^{16}$ ont confirmé cette explication. J'ai personnellement montré que la recherche empirique moderne confirme pleinement la thèse de Durkheim ${ }^{17}$. Le World Values Survey ${ }^{18}$ montre en effet que le scepticisme croît un peu partout s'agissant de la plupart des notions d'inspiration religieuse, y compris de celle de Dieu, mais que la notion d'âme apparât, elle, comme hautement résiliente.

J'aurais pu tout aussi bien prélever mes exemples dans $L a$ Division du travail social. On observe une tendance séculaire à la substitution de sanctions civiles aux sanctions pénales et à un adoucissement des peines. La cause en est que le citoyen idéal-

\footnotetext{
16 Thomas Luckmann, The Invisible Religion: The Problem of Religion in Modern Society, New York, Macmillan, 1967.

17 Raymond Boudon, Croire et savoir : penser le politique, le moral et le religieux, op. cit.; Raymond Boudon, «Les Sciences de la nature vues par les sciences sociales ", communication au colloque international et multidisciplinaire de l'Académie européenne interdisciplinaire des sciences sur Théories et modèles en sciences sociales, 28-29 novembre 2011, à paraître en 2012.

18 Ronald Inglehart et al., Human Values and Beliefs: A Cross-Cultural Sourcebook: Political, Religious, Sexual, and Economic Norms in 43 Societies: Findings from the 1990-1993 World Values Survey, Ann Arbor, The University of Michigan Press, 1998.
} 
typique tend à préférer une sanction moins lourde dès lors qu'elle apparait comme aussi efficace. Malheureusement, les manuels retiennent seulement des analyses de Durkheim sur ces questions l'idée que l'accroissement de la "densité sociale » et de la division du travail facilite l'action de ce processus.

\section{La compréhension comme conception ouverte de la rationalité}

La notion de compréhension (Verstehen) au sens de Weber est porteuse d'un troisième principe essentiel sous-jacent à la vision que Weber, Durkheim et aujourd'hui les sociologues analytiques se font de la sociologie comme science. Pour eux, les causes expliquant qu'un individu idéal-typique se comporte de la manière dont il se comporte ou croie à ce qu'il croit résident dans les raisons qui l'inspirent. Plus précisément, un individu idéaltypique endosse une croyance dès lors qu'il a l'impression que cette croyance est fondée dans un système de raisons qu'il perçoit comme acceptable, ces raisons étant dans le cas général paramétrées par le contexte. Les Sadducéens n'avaient pas les mêmes raisons que les Pharisiens de croire à l'immortalité de l'âme.

Durkheim est même plus explicite que Weber sur ce point. Il est très critique à l'endroit des auteurs de son temps qui, comme Max Müller ou Lévy-Bruhl, analysent les croyances religieuses ou magiques comme des illusions engendrées dans l'esprit des individus par des forces sociales ou psychologiques occultes et pose explicitement le postulat fondamental que les croyances persistantes ne doivent jamais être expliquées comme des illusions. Quand un jeune enfant joue avec son polichinelle, l'observateur a facilement l'impression que l'enfant nourrit l'illusion que son pantin est vivant. En réalité, l'enfant serait bien surpris si le polichinelle se mettait à le mordre. Même un chat, ironise Durkheim, comprend bien vite qu'une pelote de ficelle n'est pas une souris, puisqu'il s'en désintéresse rapidement. Durkheim aurait donc rejeté avec véhémence l'idée marxiste selon laquelle la conscience serait "fausse ", les individus étant supposés aveugles sur les raisons pour lesquelles ils font ce qu'ils font ou croient ce qu'ils croient : une idée que les sociologues relevant de 
la mouvance structuralo-marxiste traitent au contraire comme un postulat. Comme Jean-Daniel Reynaud ${ }^{19}$ l'a relevé, l'objectif de la sociologie selon Pierre Bourdieu serait de " redonner aux hommes le sens de leurs actes ", sens qui échapperait par principe aux intéressés et ne serait accessible qu'au sociologue : un postulat qui décalque l'affirmation du premier Mannheim selon laquelle seuls les intellectuels échapperaient aux illusions produites par les structures sociales. Rien de tel chez Weber et Durkheim : se concentrant normalement sur les objectifs de son action plutôt que sur ses motivations, l'individu n'est en général qu'à demi conscient des raisons qui l'inspirent. Mais des annéeslumière séparent cette remarque banale de la thèse de Marx, Mehring et Mannheim reprise par Bourdieu à travers sa notion d'habitus, qu'il prend en un sens s'écartant entièrement de la tradition classique. L'habitus ferait que les représentations de l'individu sur lui-même et sur le monde seraient obligatoirement déformées sous l'effet des structures sociales, cela entraînant une disqualification de principe des notions de bon sens et de sens commun ${ }^{20}$.

Un exemple simple tiré de Weber illustrera l'idée que les croyances doivent être expliquées par les raisons que, étant donné le contexte, les individus ont d'y adhérer. Un Occidental idéaltypique moderne est surpris que le " primitif ", comme on disait au XIX ${ }^{\mathrm{e}}$ siècle, paraisse croire en l'efficacité des rituels de pluie, mais non par le fait qu'il produise du feu en frottant deux morceaux de bois. Car, connaissant les lois de la transformation de l'énergie, l'Occidental sait que l'énergie cinétique se transforme en énergie thermique. Aussi le faiseur de feu ne le surprend-il pas, alors qu'il perçoit le comportement du faiseur de pluie comme mystérieux. Mais, s'agissant du " primitif " luimême, il n'a aucune raison de faire la même différence : il voit les pratiques du faiseur de feu et celles du faiseur de pluie comme

19 Jean-Daniel Reynaud, « Pierre Bourdieu (1930-2002) : restituer aux hommes le sens de leurs actes ", Revue française de sociologie, vol. 43, n ${ }^{\circ}$, p. I-IV, 2002. 
également fondées sur des théories que l'Occidental perçoit comme " magiques".

Dans cet exemple et dans toutes ses analyses, Weber propose de substituer une conception cognitive de la rationalité à la conception instrumentale courante. L'exemple est en d'autres termes porteur de l'idée cruciale selon laquelle comprendre un acte, une croyance ou un comportement individuels signifie déterminer les raisons qui les ont inspirés dans l'esprit de l'individu, étant admis que le déchiffrement desdites raisons implique que l'observateur soit conscient des traits caractérisant le contexte social et cognitif dans lequel l'individu est plongé.

J'ai personnellement proposé de formaliser la théorie de la rationalité de Weber et de l'appeler théorie de la rationalité ordinaire, afin de la distinguer de la théorie la plus couramment mise en ouvre aujourd'hui dans les sciences sociales : la théorie $d u$ choix rationnel. La différence entre les deux est que la seconde introduit l'idée selon laquelle les raisons qui mobilisent les individus seraient essentiellement égoïstes et instrumentales, tandis que selon la première elles peuvent être aussi impersonnelles plutôt qu'égoïstes et cognitives plutôt qu'instrumentales. Ainsi, les raisons expliquant que les Occidentaux perçoivent le comportement des faiseurs de pluie comme étrange, mais non celui des faiseurs de feu sont cognitives et non instrumentales, impersonnelles et en aucune façon égoïstes.

La compétence la théorie du choix rationnel étant limitée à l'explication des moyens mis en ouvre par les individus pour atteindre leurs objectifs, elle est vouée à expliquer les objectifs, les valeurs et les croyances des individus en évoquant des causes conjecturales opérant sans intervention de leur esprit. Les théoriciens du choix rationnel qualifient généralement ces causes de cadres (frame, framework). Mais on repère le même schème explicatif sous divers habillages, comme mentalité primitive chez Lévy-Bruhl ou habitus au sens de la mouvance structuralomarxiste. Certains tentent de déduire ces causes conjecturales de mécanismes psychologiques, sociologiques ou biologiques eux- 
mêmes conjecturaux. Karl Popper ${ }^{21}$ a argué pour cette raison que, tant que les sciences sociales utiliseraient des notions de ce genre, elles auraient des pieds d'argile. Les incertitudes de la sociologie proviennent en effet en grande partie de ce que, en acceptant trop facilement l'idée que les structures sociales déterminent le comportement, elle tombe dans le sociologisme.

Ce sociologisme est le dénominateur commun de plusieurs théories influentes. Il sous-tend le structuralisme, la mémétique, le "programme fort " de la sociologie des sciences selon lequel, non seulement les sujets qu'aborde l'homme de science, mais les vérités auxquelles il croit parvenir seraient l'effet des structures sociales, les théories radicales du genre, selon lesquelles les structures sociales expliqueraient la domination de l'individu de sexe masculin, laquelle entraînerait à son tour toutes sortes d'effets, dont une prévalence statistique de l'hétérosexualité. Loin d'échapper à la conception déterministe selon laquelle tout comportement serait l'effet des structures sociales, le constructivisme vise à montrer que les constructions qu'il se fait fort de " déconstruire " sont l'effet des structures sociales.

On décèle l'importance de la tentation sociologiste en sociologie à ce que bien des sociologues ont tendance à définir leur discipline comme la science des effets des structures sociales, alors que toutes les autres sciences se définissent, non par un facteur explicatif, mais par une classe de phénomènes à expliquer. Le sociologisme s'inspire le plus souvent de motivations politiques, qu'il s'agisse de faire descendre la science de son piédestal et d'atténuer par là son influence, d'imposer certaines politiques scolaires ou de défendre telle ou telle minorité. C'est pourquoi Michael Tåhlin ${ }^{22}$ se félicite du pluralisme académique dont témoigne la sociologie, mais regrette qu'elle ne fasse pas état du même pluralisme en matière idéologique. Selon lui, l'intérêt de la sociologie varie en raison inverse de la charge idéologique

21 Karl Popper, "The Myth of the Framework ", dans Freeman, R. Edward (dir.), The Abdication of Philosophy. Philosophy and the Public Good, La Salle (Ill), Open Court, 1976, p. 23-48.

22 Michael Tåhlin, "Laissez-moi ma liberté. Pour une sociologie pluraliste ", Commentaire, hiver 2011-2012, 1077-1082. 
qu'elle véhicule. Cela dit, la tentation idéologique des sciences humaines est de tous les temps. La grandeur de Tocqueville tient pour une part à sa volonté de neutralité scientifique ${ }^{23}$. Or sa pensée s'imposa beaucoup plus difficilement en son temps que celle d'un Michelet.

Une distinction claire doit être introduite entre les variables dispositionnelles comme celles qu'introduisent les notions de mentalité primitive ou d'habitus au sens structuralo-marxiste et les paramètres décrivant le contexte dans lequel se déploie le comportement. Que les "primitifs " ne connaissent pas les lois de la transformation de l'énergie est un paramètre distinguant leur contexte de celui de l'observateur. Que les premiers Chrétiens ne fassent pas de distinction entre le naturel et le surnaturel est un paramètre distinguant le contexte qui était le leur du contexte créé par l'idée que les phénomènes naturels sont gouvernés par des lois. De tels paramètres sont des données de fait, alors que les variables dispositionnelles sont des constructions conjecturales et ad hoc. La célèbre "baignoire " de Coleman ${ }^{24}$ ignore cette distinction cruciale et confond les deux catégories dans la catégorie des " attitudes et dispositions individuelles ». Il n'est évidemment pas question de nier l'existence de dispositions. Mais leurs effets sur le comportement sont hautement imprévisibles. Comme Simmel ${ }^{25}$ l'a souligné, une éducation autoritaire peut produire une personnalité libérale autant qu'une personnalité autoritaire. Les études empiriques montrent abondamment que les relations entre dispositions et comportements sont faibles et variables d'une étude à l'autre. La grande étude classique d'Adorno sur $\mathrm{La}$ Personnalité autoritaire est un exemple canonique de recherche visant à montrer le caractère déterminant de certaines dispositions sur certains comportements. Elle a été accueillie dès sa parution par des commentaires largement dubitatifs. Une grande leçon à tirer des analyses de Max Weber est qu'il ne mobilise pratiquement jamais de variables dispositionnelles pour expliquer

23 Raymond Boudon, Tocqueville aujourd'hui, Paris, Odile Jacob, 2005.

24 James Coleman, Foundations of Social Theory, Cambridge (MA), Harvard University Press, 1990.

25 Georg Simmel, Die Probleme der Geschichtsphilosophie, op. cit. 
un comportement. La force de ses analyses tient en grande partie à ce qu'il adopte, comme Durkheim, une attitude d'agnosticisme à l'endroit des dispositions. En fait, les variables dispositionnelles ont été introduites dans la boîte à outils du sociologue sous l'influence du béhaviorisme, un mouvement de pensée qui se présenta comme capable de faire de la psychologie une science dure et qui affecta toutes les sciences humaines. Cela entraîna par une ironie de l'histoire la légitimation dans les sciences sociales des explications raillées par Molière, du type "l'opium fait dormir parce qu'il a une vertu dormitive ".

La théorie de la rationalité ordinaire que je propose de tirer des travaux de Weber repose finalement sur un principe de base que je propose d'appeler principe de l'équilibre cognitif. Il énonce qu'un individu croit que $\mathrm{X}$ est vrai, acceptable, bon, légitime, etc., à partir du moment où il a l'impression que ce jugement repose sur un système de raisons acceptables.

À côté du singularisme méthodologique et de l'individualisme méthodologique, le principe de l'équilibre cognitif est finalement le troisième principe que je vois comme définissant implicitement le paradigme utilisé par les pères fondateurs et tout autant par beaucoup de sociologues modernes : un paradigme qui occupe le cœur de la sociologie analytique.

\section{Réseaux et Generalized others}

Le troisième principe exclut le modèle de l'acteur social solipsiste du fait qu'un individu tend à percevoir les raisons fondant ses croyances comme valides seulement s'il a l'impression qu'elles ont vocation à être partagées. J'ai proposé de qualifier ces raisons de transsubjectives. L'idée selon laquelle les raisons motivant une croyance dans l'esprit de l'individu ne lui apparaissent comme valides que s'il a l'impression qu'elles devraient être partagées propose une définition formelle de la célèbre notion des generalized others de George Mead ${ }^{26}$. Le principe de l'équilibre cognitif introduit ainsi une différence essentielle entre la théorie $d u$ choix

26 George Herbert Mead, L'Esprit, le soi, la société, Paris, Presses universitaires de France, 2006 [1934]. 
rationnel, qui véhicule une vision solipsiste de l'acteur social, et la théorie de la rationalité ordinaire, qui restaure sa qualité d'être social.

Remarque incidente. Le thème des réseaux est aujourd'hui florissant dans la recherche sociologique. Mais les réseaux sociaux y sont souvent traités de façon descriptive ou mécanique, alors qu'une symbiose de la notion de réseau avec la théorie de la rationalité ordinaire produirait un surcroît d'intelligibilité. Tocqueville a écrit que "nous croyons un million de choses sur la foi d'autrui ». En effet, dans bien des cas, dès qu'un ami ou un voisin nous paraît crédible, nous choisissons de nous laisser influencer par son jugement sur les sujets sur lesquels il nous semble plus compétent ou mieux informé. Le célèbre two-step flow of communication de Lazarsfeld ${ }^{27}$ opérationnalise et illustre cette idée. Elle est centrale chez Coleman et al. ${ }^{28}$ ou Manzo ${ }^{29}$. En un mot, l'activation par l'individu de ses réseaux peut être rendue intelligible à partir de la théorie de la rationalité ordinaire.

\section{Illustrations}

Dans ce qui suit, je tenterai de suggérer par des exemples relevant de plusieurs chapitres que le paradigme des trois principes caractérisant implicitement la sociologie analytique, les principes du singularisme méthodologique, de l'individualisme méthodologique et de l'équilibre cognitif constitue un cadre théorique au sein duquel toutes sortes de phénomènes intéressant les sciences sociales peuvent être expliqués et ont de fait été expliqués.

Je considérerai successivement des exemples où les phénomènes sociaux à expliquer sont des croyances représentationnelles, des croyances normatives, des tendances de long et moyen terme et des solutions pratiques à des dilemmes classiques engendrés par les situations d'interaction, comme le dilemme du prisonnier. Je

27 Elihu Katz et Paul Lazarsfeld, Personal Influence, Glencoe (Ill), The Free Press 1955 et Londres, Transaction, 2006.

28 James Coleman, Elihu Katz et Herbert Menzel, Medical Innovation, Indianapolis, Bobbs-Merrill, 1966.

29 Gianluca Manzo, La Spirale des inégalités. Choix scolaires en France et en Italie au XXe siècle, Paris, Presses de l'Université de Paris-Sorbonne, 2009. 
chercherai aussi à montrer par ces exemples que, bien que la notion de sociologie analytique soit nouvelle, elle verse un vieux vin dans des bouteilles neuves, puisqu'elle revitalise essentiellement les principes plus ou moins implicitement utilisés par les sociologues classiques, notamment par Max Weber et Emile Durkheim.

\section{Croyances représentationnelles}

Un exemple tiré de Weber illustrera l'importance pour l'explication des croyances représentationnelles des trois principes logés à la racine de la sociologie analytique.

Pourquoi, se demande Max Weber ${ }^{30}$, les officiers et les fonctionnaires romains sont-ils attirés par les cultes monothéistes importés du Moyen-Orient comme le culte de Mithra puis le christianisme, alors que les paysans y sont durablement hostiles et restent fidèles à la religion polythéiste traditionnelle? L'hostilité de ces derniers au christianisme était si profonde que le mot paganus (paysan) a fini par désigner les non-croyants, les païens.

S'agissant des paysans, Weber explique que l'imprévisibilité des phénomènes naturels qui domine leur activité leur paraît incompatible avec l'idée que l'ordre des choses puisse être soumis à une volonté unique obligatoirement dotée d'un minimum de cohérence. Ils acceptèrent plus facilement le christianisme dès lors qu'une foule de saints lui conféra un caractère polythéiste davantage compatible avec leur expérience.

En un mot, les paysans romains fondent leurs croyances sur un système de raisons qui leur paraissent valides. Il en va de même des officiers et des fonctionnaires : avec son côté hiérarchique et ses rites d'initiation impersonnels, le culte de Mithra leur paraissait refléter fidèlement l'organisation de l'Empire romain dans un registre symbolique. Cela devait par la suite faciliter la diffusion du christianisme parmi eux. La contextualité de ces croyances ordinaires n'implique pas qu'elles soient dépourvues de raisons.

$30 \quad$ Max Weber, Gesammelte Aufsätze zur Religionssoziologie, Munich, Mohr, 1986 [1920]. 


\section{Les croyances en l'efficacité des rituels magiques}

J'utiliserai un exemple tiré de Durkheim comme seconde illustration du paradigme à trois principes : celui de son explication de l'adhésion aux croyances magiques. Il a l'avantage d'être particulièrement brillant, et aussi de montrer que Durkheim et Weber appliquent les mêmes principes. La difficulté est que les écrits réflexifs de Weber coïncident avec ses analyses empiriques, alors que ceux de Durkheim sont beaucoup plus confus. Incidemment, Parsons ${ }^{31}$ a bien perçu la convergence de fond entre les deux auteurs puisqu'il tente dans sa Structure of Social Action de fonder la sociologie sur une synthèse essentiellement de leurs travaux.

Le thème de l'adhésion aux croyances magiques a de surcroît l'intérêt qu'il a inspiré un ensemble de trois explications canoniques, dont l'une domine franchement les autres. Premier type de théorie : Wittgenstein ${ }^{32}$ avance que les praticiens des rituels magiques, par exemple des rituels de pluie, ne croiraient pas réellement en l'efficacité de leurs rituels. Ils manifesteraient seulement en les accomplissant leur désir de voir tomber la pluie. Deuxième type de théories : s'inspirant d'Auguste Comte, LévyBruhl $^{33}$ veut que les croyances magiques proviennent de ce que dans les premiers âges de l'humanité les hommes aient été peu sensibles à la contradiction et aient confondu les relations de similarité et de causalité. C'est pourquoi ils ont cru par exemple qu'en blessant une poupée représentant son ennemi on pouvait atteindre son ennemi lui-même. Beattie ${ }^{34}$, Needham ${ }^{35}$ et Sahlins ${ }^{36}$ ont suivi Lévy-Bruhl. Ils veulent que la rationalité soit dépen-

31 Talcott Parsons, The Structure of Social Action: A Study in Social Theory with Special Reference to a Group of Recent European Writers, New York, McGrawHill, 1937. Nouvelle édition, Glencoe (Ill), The Free Press, 1949.

32 Ludwig Wittgenstein, "Bemerkungen über Frazer's, The Golden Bough ", Synthese, no 17, 1967, p. 233-253.

33 Lucien Lévy-Bruhl, La Mentalité primitive, Paris, Presses universitaires de France, 1960 [1922].

34 John Beattie, Other Cultures, Londres, Cohen \& West, 1964.

35 Rodney Needham, Belief, Language and Experience, Oxford, Blackwell, 1972.

36 Marshall, Sahlins, Culture and Practical Reason, Chicago, University of Chicago Press, 1976. 
dante de la culture. Troisième type de théorie : Durkheim ${ }^{37}$, Weber $^{38}$ ou aujourd'hui Robin Horton ${ }^{39}$, veulent que les croyances magiques soient le produit de la rationalité ordinaire.

La théorie de Wittgenstein est incompatible avec nombre d'observations. Car, comme l'a montré Horton, les Africains continuent de croire fermement en l'efficacité de la magie après leur conversion au christianisme. Lorsqu'on leur demande pourquoi, ils expliquent qu'il manque au christianisme de proposer des recettes utiles pour la conduite de la vie quotidienne. De surcroît, ils rejettent sans hésitation l'idée que les rituels magiques soient dépourvus d'effets.

S'agissant de la théorie de Lévy-Bruhl et de ses variantes, qui ont en commun de voir la rationalité comme culturellement variable, ils introduisent une conjecture lourde qui se présente comme ad hoc et circulaire, sa crédibilité étant exclusivement fondée sur les effets qu'elle est censée expliquer.

La théorie proposée par Durkheim de la magie blanche est l'une de ses théories particulières les plus remarquables, bien qu'elle soit présentée dans des passages éparpillés des Formes élémentaires de la vie religieuse $e^{40}$.

Selon cette théorie, il faut d'abord reconnaitre que le savoir du "primitif " n'est pas celui de l'Occidental. Il n'a pas, comme lui, été initié à la méthodologie de l'inférence causale et il n’a aucune raison de maitriser les principes de la biologie ou de la physique.

La conduite de la vie quotidienne, mais aussi la production agricole, la pêche ou l'élevage supposent toutes sortes de savoirfaire. Pour une part, ceux-ci sont tirés dans les sociétés traditionnelles, comme chez nous, de l'expérience. Mais les données de l'expérience ne peuvent prendre sens que sur un fond de représentations théoriques de la vie, de la croissance, de la mort, de la nutrition et, de manière générale, des processus vitaux. Ces

\footnotetext{
37 Émile Durkheim, Les Formes élémentaires de la vie religieuse, Paris, Presses universitaires de France, 1979 [1912].

38 Max Weber, Économie et société, Paris, Plon, 1971 [1922].

39 Robin Horton, Patterns of Thought in Africa and the West, Cambridge, Cambridge University Press, 1993.

40 Voir Raymond Boudon, La Sociologie comme science, op. cit.
} 
représentations ne pouvant pas être directement déduites de l'expérience, le " primitif " les tire normalement du corpus de savoir disponible et tenu pour légitime dont il dispose. Car "l'essentiel [est] de ne pas laisser l'esprit asservi aux apparences sensibles, mais, au contraire, de lui apprendre à les dominer et à rapprocher ce que les sens séparent $»^{41}$. Dans le cas des sociétés qu'envisage Durkheim, ce sont les doctrines religieuses qui fournissent des explications du monde permettant de coordonner les données de l'expérience sensible. Ces doctrines jouent donc dans les sociétés traditionnelles le rôle de la science dans les sociétés modernes, au sens où elles représentent le corpus de savoir légitime. Car les religions représentent une première forme de l'explication du monde : "Le grand service que les religions ont rendu à la pensée est d'avoir construit une première représentation de ce que pouvaient être ces rapports de parenté entre les choses $^{42}$ ".

Quant aux croyances magiques, elles sont des recettes que le " primitif " tire de la "biologie " qu'il construit à partir des doctrines en vigueur dans sa société.

Une question se pose alors : les recettes magiques manquent d'efficacité; les croyances et les attentes qui se développent à partir de théories sans fondement objectif tendent à être contredites par le réel. Comment se fait-il alors que leur crédibilité se maintienne, en dépit du postulat de la fragilité des illusions que défend Durkheim? Il répond à cette objection qu'il se fait à luimême par une série d'arguments efficaces.

Tout d'abord, il est difficile de tirer une conclusion immédiate d'une situation où une croyance causale se trouve contredite par l'observation. Les savants eux-mêmes continuent couramment d'accorder leur confiance à une théorie contredite par les faits. Anticipant sur des développements de la philosophie moderne des sciences dus notamment à Duhem, puis Kuhn, Quine et Lakatos, Durkheim avance que les hommes de science ont de sérieuses raisons de ne pas abandonner une théorie contredite par

\footnotetext{
41 Émile Durkheim, Les Formes élémentaires de la vie religieuse, op. cit., p. 340. 42 Ibid., p. 340.
} 
les faits. Ils peuvent en effet toujours espérer que la contradiction opposée à une théorie par tel fait résulte d'un élément secondaire et qu'une modification mineure de la théorie se révélera capable de l'éliminer. Au total, il est souvent plus rationnel de conserver une théorie contredite par les faits et de tenter de l'amender que de la repousser. "Quand une loi scientifique a pour elle l'autorité d'expériences nombreuses et variées, il est contraire à toute méthode d'y renoncer trop facilement sur la découverte d'un fait qui paraît la contredire. [...]. Or l'Australien ne procède pas autrement quand il attribue l'insuccès d'un Intichiuma à quelque maléfice $[. . .]^{43}$ ".

$\mathrm{Kuhn}^{44}$ confirme sans le savoir les remarques de Durkheim lorsqu'il montre que Priestley avait toutes les raisons du monde de s'accrocher à la théorie du phlogistique, aujourd'hui définitivement discréditée. Lakatos ${ }^{45}$ confirme, lui aussi, la théorie de Durkheim par une parabole célèbre : une théorie contredite par les faits peut conserver la confiance des savants pendant des siècles, car on peut faire l'hypothèse qu'un aménagement de la théorie éliminera la contradiction et qu'il est toujours possible de créer des hypothèses auxiliaires crédibles dont la vérification peut être tributaire d'innovations techniques lentes à venir.

Exactement comme les hommes de science, nous dit Durkheim, les magiciens imaginent sans difficulté des hypothèses auxiliaires pour expliquer pourquoi leur théorie a échoué : les rituels n'ont pas été accomplis comme il le fallait; les dieux étaient de mauvaise humeur ce jour-là, à moins que des facteurs non identifiés n'aient perturbé l'expérience.

La réalité peut, de surcroît, confirmer des croyances fausses. En effet, les rituels destinés à faire tomber la pluie ou à faciliter la reproduction des troupeaux sont effectués à l'époque où les

$43 \quad$ Ibid., p. 515.

44 Thomas S. Kuhn, The Structure of Scientific Revolutions, Chicago, University Chicago Press, 1962.

45 Imre Lakatos, "Falsification and the Methodology of Scientific Research Programs ", dans Imre Lakatos et Alan Musgrave (dir.), Criticism and the Growth of Knowledge, vol. 4, Londres, Cambridge University Press, 1965, p. 91-195. 
récoltes ont besoin de pluie et par conséquent où elle a plus de chances de tomber ou à l'époque où les animaux s'accouplent. Ainsi, la croyance en une relation de causalité fausse peut être confirmée par l'existence de corrélations qui, bien que fallacieuses, sont avérées ${ }^{46}$.

On peut ajouter deux arguments à ceux de Durkheim. En premier lieu, la critique d'une relation causale n'est pas toujours facile. Elle suppose en effet que soient réalisées des conditions quasi-expérimentales qui ne le sont normalement pas. De plus, une telle critique peut n'être possible que grâce à la mobilisation d'instruments statistiques dont le "primitif " ne dispose pas.

En second lieu, pour que la foi en une théorie s'estompe, celle-ci doit pouvoir être remplacée par une théorie concurrente. Or les sociétés traditionnelles sont caractérisées par le fait que les interprétations du monde qu'elles endossent sont faiblement évolutives. Le marché de la construction des théories y est peu actif, et il est normalement moins concurrentiel s'agissant des théories religieuses que des théories scientifiques.

Durkheim suggère au total que les croyances collectives que l'on observe dans les sociétés traditionnelles et que l'on perçoit comme magiques ne sont pas d'une essence autre que les croyances collectives qu'on observe dans les nôtres. Mais, comme le développement de la science a frappé d'une obsolescence définitive certaines de ces croyances, quand nous constatons que quelqu'un continue d'y souscrire, nous avons tendance à le considérer comme " irrationnel ».

En fait, suggère Durkheim, ces croyances magiques sont des conjectures que le "primitif " forge à partir du savoir qu'il considère légitime, exactement comme nous adhérons nousmêmes, à partir du savoir qui est le nôtre, à toutes sortes de relations causales dont les unes sont fondées, mais dont les autres sont tout aussi fragiles ou illusoires que celles des "primitifs". On a longtemps cru que le stress était la cause de l'ulcère à l'estomac jusqu'à ce que l'on démontre qu'il est d'origine bactérienne. Et l'on ne compte plus les recettes magiques que les experts

$\overline{46}$ Émile Durkheim, Les Formes élémentaires de la vie religieuse, op. cit., p. 516. 
proposent à l'homme moderne pour perdre du poids, assurer la repousse de ses cheveux ou renforcer sa virilité.

Ces croyances s'expliquent, comme les croyances des "primitifs ", par le fait qu'elles font sens pour nous, en d'autres termes, que nous avons des raisons d'y adhérer.

La théorie de Durkheim est solide, en premier lieu, parce qu'elle se compose de propositions qui sont toutes, prises individuellement, aisément acceptables. Toutes énoncent des données factuelles ou se réfèrent à des lois psychologiques avérées comme "l'être humain désire survivre ", " on préfère chercher à amender une théorie qui a fait ses preuves plutôt que de la jeter aux orties à sa première défaillance ", " on tend à voir dans une corrélation une présomption de causalité ". En second lieu, la théorie de Durkheim est congruente avec des faits établis bien après lui. Elle explique en effet, non seulement le phénomène de la magie lui-même, mais ses variations dans le temps et dans l'espace. Armé de cette théorie, on n'a aucune peine à comprendre que les pratiques magiques ne soient en aucune façon uniformément distribuée à travers les sociétés primitives. Elles sont par exemple peu répandues dans la Chine et dans la Grèce classiques. Cela est dû à ce que les doctrines religieuses en vigueur dans ces sociétés insistent sur les régularités qui président au fonctionnement du cosmos, ne laissant guère de place aux forces capricieuses que la pensée magique met en œuvre.

De même, il est facile de comprendre grâce à la théorie durkheimienne que la magie blanche comme la magie noire - la sorcellerie - soient peu répandues en Europe à la fin du Moyen Âge : l'Europe est alors dominée par la pensée aristotélicienne dont le caractère empiriste n'encourage guère à expliquer le monde par des forces magiques ${ }^{47}$. Les pratiques magiques qu'on observe alors relèvent de la magia naturalis : ce sont des recettes techniques issues de conjectures relatives aux phénomènes naturels qui ne se distinguent guère dans leur principe des conjectures du savant.

47 Guy Bechtel, La Sorcière et l'Occident, Paris, Plon, 1997. Keith Thomas, Religion and the Decline of Magic: Studies in Popular Beliefs in Sixteenth and Seventeenth Century England, London, Penguin Books, 1973. 
En revanche, la Renaissance s'accompagne d'un discrédit de l'aristotélisme, auquel se substitue une vogue du néo-platonisme. Car les " humanistes » du XVI ${ }^{\mathrm{e}}$ siècle cherchent à manifester leur modernité par une rupture avec une doctrine associée à un Moyen Âge perçu comme dépassé. Or le néo-platonisme est une philosophie beaucoup plus accueillante que l'aristotélisme aux explications du monde à partir de forces mystérieuses. Alors que les mythes sont pour Aristote des explications symboliques du monde qu'il faut se garder de prendre au pied de la lettre, ils représentent pour Platon des théories explicatives au sens fort. Avec le néo-platonisme s'impose l'idée que les phénomènes naturels s'expliquent par des forces occultes. Apparaît alors la magia diabolica, qui n'a rien à voir avec la magia naturalis, et qui explique notamment le développement des procès en sorcellerie.

La théorie de Durkheim permet ainsi de comprendre que la bouffée prolongée de magie et de sorcellerie qui apparaît en Europe au $\mathrm{XVI}^{\mathrm{e}}$ siècle, pour se prolonger jusqu'au milieu du XVIII ${ }^{\mathrm{e}}$ siècle, soit localisée dans la partie la plus moderne et la plus développée du continent. Elle est en effet beaucoup plus intense en Italie du Nord et en Allemagne du Sud qu'en Italie du Sud ou en Espagne. Au total, le cadre théorique durkheimien permet d'expliquer facilement cette combinaison à première vue surprenante de modernité et d' "irrationalisme " qui caractérise le début des temps modernes ${ }^{48}$.

Il permet également d'expliquer facilement des énigmes voisines, comme la persistance des croyances "irrationnelles " chez les pionniers des sciences modernes. La théorie durkheimienne explique par exemple que l'alchimie ait joué un grand rôle dans la genèse de la chimie, ou que Newton ait été un alchimiste fervent.

Elle permet aussi de comprendre, ainsi que cela a été relevé par Hubert et Mauss ${ }^{49}$, pourquoi les pratiques magiques sont plus

\footnotetext{
48 Voir Raymond Boudon, La Sociologie comme science, op. cit. et Croire et savoir : penser le politique, le moral et le religieux, op. cit.

49 Henri Hubert et Marcel Mauss, "Esquisse d'une théorie générale de la magie ", L’Année sociologique 7, 1902-1903, p. 1-146. Repris dans Marcel Mauss, Sociologie et anthropologie, précédé d'une «Introduction à l'œuvre de
} 
développées, toutes choses égales d'ailleurs, dans les sociétés où l'activité économique dominante est aléatoire, comme dans les sociétés de pêche.

La théorie de Durkheim explique les variations des croyances et des pratiques magiques dans le temps et dans l'espace, et aussi pourquoi science et magie cohabitent encore si aisément de nos jours. Ce point est à rapprocher du fait que Durkheim n'éprouvait aucune difficulté à comprendre que la modernité s'accompagne, non seulement d'une vitalité des sentiments religieux, mais même d'une vitalité des traditions religieuses les plus anciennes ${ }^{50}$. C'est pourquoi il récuse violemment ceux qui veulent voir dans «le catholique éclairé d'aujourd'hui, une sorte de sauvage attardés ${ }^{51}$.

La théorie de Durkheim est donc bien supérieure aux deux autres types de théories, celles que défendent Lévy-Bruhl, Wittgenstein et leur continuateurs, parce qu' elle introduit exclusivement des propositions empiriques irrécusables et des lois psychologiques avérées. Cet exemple a ainsi l'intérêt de souligner que le fait d'imputer le comportement d'autrui à des raisons n'implique aucun subjectivisme de la part de l'analyste. Reconstruire les raisons d'autrui revient en fait à élaborer une théorie qui, encore une fois, ne comporte que des propositions empiriques et des lois psychologiques incontestables.

Les sociologues analytiques parlent de mécanismes pour qualifier des propositions telles que " en général, les individus préferent survivre " ou " on préfere chercher à amender une théorie qui a fait ses preuves ". Comme Demeulenaere ${ }^{52}$ le suggère, on peut aussi utiliser le concept classique de $l o i$.

Marcel Mauss » par Claude Lévi-Strauss, Paris, Presses Universitaires de France, 1950, p. 1-141.

50 Boudon, Raymond, Croire et savoir : penser le politique, le moral et le religieux, op. cit.

51 Emile Durkheim, Les Formes élémentaires de la vie religieuse, op. cit., p. 460.

52 Pierre Demeulenaere, «Mécanismes, causalité, régularités de comportements et normes sociales", op. cit. 


\section{Croyances normatives}

J'illustrerai maintenant l'importance du paradigme à trois règles qui définit la sociologie analytique pour l'explication des croyances normatives. J'utiliserai d'abord un exemple emprunté à Adam Smith.

On imagine souvent qu'Adam Smith souscrit aveuglément à la version instrumentale de la rationalité. L'exemple qui suit montre qu'il sollicite discrètement dans La Richesse des nations une notion développée dans sa Théorie des sentiments moraux, celle du spectateur impartial. Le spectateur impartial, c'est l'individu qui serait par hypothèse en situation de mettre entre parenthèses ses passions et ses intérêts et de faire appel à son seul bon sens: un individu obéissant en d'autres termes à la rationalité ordinaire.

Cet exemple revêt une portée particulière, car il esquisse un dépassement de la théorie pratique de Kant. Smith y montre en effet que, à la différence de la raison pratique, la rationalité axiologique ordinaire, loin de devoir s'en tenir à des préceptes généraux, peut expliquer les jugements normatifs et généralement appréciatifs émis dans des contextes divers et rendre compte par là de la variabilité des croyances axiologiques dans le temps et dans l'espace.

Adam Smith ${ }^{53}$ se demande pourquoi les Anglais de son temps considèrent comme légitime que les mineurs soient davantage payés que les soldats. Comment expliquer cette croyance collective? Sa réponse : la plupart des Anglais n'étant ni mineurs ni soldats et n'étant par suite pas directement concernés par la question sont dans la position du spectateur impartial. Leur sentiment est donc fondé sur un système de raisons de bon sens qui tendent à être partagées.

Ce système est le suivant : le salaire est la rémunération d'un service rendu. À service équivalent, les salaires doivent être équivalents. Dans la valeur d'un service rentrent divers éléments : notamment la durée d'apprentissage qu'il implique et les risques

53 Adam Smith, An Inquiry into the Nature and Causes of the Wealth of Nations, $7^{\mathrm{e}}$ édition, Londres, Strahan and Cadell, 1976 [1793], Ch X. 
auxquels il expose celui qui le rend. Dans le cas du mineur et du soldat, les durées d'apprentissage sont comparables et, dans les deux cas, l'individu risque sa vie. Mais les activités en question se distinguent par d'autres traits. Le soldat garantit l'existence de la nation; le mineur exerce une activité orientée vers la production de biens matériels moins fondamentaux que l'indépendance nationale. En outre, la mort du mineur fait partie des risques du métier : elle est un accident. Le soldat, lui, s'expose à la mort pour le salut de la patrie : sa mort est un sacrifice, que son engagement soit volontaire ou non. Il est donc habilité à recevoir le respect, la gloire et les symboles de reconnaissance qui sont dus à celui qui met sa vie en jeu pour la collectivité.

Il résulte de ces arguments et du principe de l'égalité entre contributions et rétributions que, ne pouvant recevoir ces marques symboliques de reconnaissance et accomplissant un travail aussi pénible et aussi risqué, le mineur doit recevoir en une autre monnaie les récompenses qu'il ne peut recevoir en gloire. C'est pourquoi il doit être mieux payé que le soldat.

Cette analyse montre que le consensus sur la proposition que les mineurs doivent être davantage payés que les soldats est la conséquence de données de fait et de principes facilement acceptables. C'est aussi sous l'effet de raisons que l'exécuteur public, explique Adam Smith, doit recevoir un bon salaire : sa qualification est minime, mais il exerce "le plus répugnant de tous les métiers".

Michael Walzer ${ }^{54}$ 'sinterroge, lui aussi, sur des sentiments moraux dont on peut présumer qu'ils sont partagés et en propose, lui aussi, une explication judicatoire, pour employer le terme par lequel Montaigne désignait les explications fondées sur des raisons ayant vocation à être partagées. Pourquoi, se demande-t-il, le public considère-t-il la conscription comme acceptable s'agissant des militaires, mais non des mineurs. La réponse est, ici encore, que l'activité du soldat est plus centrale que les activités de caractère économique. Si l'on appliquait la conscription à telle

54 Michael Walzer, Sphères de justice. Une défense du pluralisme et de l'égalité, Paris, Seuil, 1997. 
activité économique particulière, on serait en droit de l'appliquer à toutes : cela reviendrait à la limite à justifier le travail forcé.

On explique aussi par des raisons fortes, peut-on ajouter dans le même esprit, le fait qu'on accepte facilement que les conscrits puissent être affectés à des tâches de police urbaine s'ils s'y portent candidats ou que l'armée soit chargée de ramasser les poubelles lorsqu'une grève des éboueurs se prolonge, entraînant des risques pour la santé publique. Mais on n'admettrait pas que la police urbaine ou le ramassage des ordures soient des activités normalement assurées par la conscription.

Ces évidences morales sont fondées sur des raisons. Elles se présentent comme les corollaires de principes que le spectateur impartial peut facilement considérer comme acceptables.

En résumé, la variation contextuelle des croyances morales n'implique aucun relativisme. Montaigne ne peut être mobilisé pour justifier le relativisme, car les croyances morales ne peuvent être assimilées à des coutumes. Hume non plus, car elles sont fondées, non sur l'habitude, mais sur des raisons qu'approuverait le spectateur impartial.

De façon générale, la fiction du spectateur impartial est indispensable à l'analyse de maintes questions majeures des sciences sociales : celles notamment des relations entre égalité et équité, des raisons d'être des phénomènes de consensus ou des phénomènes d'évolution des idées et des institutions sur le moyen et le long terme.

\section{Égalité et équité}

Selon diverses enquêtes, le public distingue clairement entre plusieurs sortes d'inégalités et n'assimile inégalité et injustice que dans des cas bien déterminés. C'est qu'il y a sous les sentiments de justice ou d'injustice suscités par telle ou telle forme d'inégalités des raisons ayant de bonnes chances d'être approuvées par le spectateur impartial.

Ainsi, les inégalités fonctionnelles ne sont pas perçues comme injustes : le public admet que les rémunérations soient indexées sur le mérite, les compétences ou l'importance des services ren- 
dus. Ne sont pas non plus perçues comme injustes les inégalités résultant du libre choix des individus. Les rémunérations des vedettes $\mathrm{du}$ sport ou du spectacle sont considérées comme excessives mais non comme injustes par la raison qu'elles résultent de l'agrégation de demandes individuelles de la part de leur public. En principe, il faut que, à contribution identique, la rétribution soit identique. Mais on ne considère pas comme injuste que deux personnes exécutant les mêmes tâches soient rémunérées différemment selon qu'elles appartiennent à une entreprise ou à une région florissante ou non. On ne considère pas non plus comme injustes des différences de rémunération concernant des activités incommensurables. Ainsi, il est difficile de déterminer si le climatologue doit être plus ou moins rémunéré que l'huissier de justice. On ne considère pas non plus comme injustes les inégalités dont on ignore l'origine et dont on ne peut par suite déterminer si elles sont fonctionnelles ou non.

On considère en revanche comme injustes les inégalités perçues comme des privilèges. Ainsi, les parachutes dorés que certains chefs d'entreprise se font octroyer par un conseil à la composition duquel ils ne sont éventuellement pas étrangers sont particulièrement mal perçus, surtout lorsque leurs contributions ne sont guère visibles.

Le voile d'ignorance évoqué par John Rawls ${ }^{55}$ dans sa théorie de la justice introduit une fiction analogue à celle du spectateur impartial d'Adam Smith. Elle a inspiré divers travaux qui permettent de préciser la notion de rationalité axiologique.

\section{L'impôt sur le revenu}

Les sociétés démocratiques ont longtemps débattu dans le passé de la pertinence de l'impôt sur le revenu. On en a contesté le principe. Lorsqu'on l'a accepté, on l'a voulu d'abord proportionnel. Aujourd'hui, un consensus général s'est établi sur l'idée que l'impôt sur le revenu est une bonne chose, et qu'il doit être modérément progressif. Si un consensus a fini par s'établir sur ce

55 John Rawls, A Theory of Justice, Cambridge (MA), Harvard University Press, 1971. 
point, c'est qu'on discerne au fondement de cette conviction collective un système de raisons qu'approuverait le spectateur impartial.

Les sociétés modernes sont grossièrement composées, comme Tocqueville l'avait déjà relevé, de trois classes sociales. Elles entretiennent entre elles des relations à la fois de coopération et de conflit. Il s'agit : des riches, qui disposent d'un surplus significatif éventuellement convertible en pouvoir politique ou social; de la classe moyenne, qui ne dispose que d'un surplus limité, insuffisant pour être converti en pouvoir; des pauvres ${ }^{56}$.

La cohésion sociale et la paix sociale du côté des effets attendus et le respect de la dignité de tous du côté des principes impliquent que les pauvres soient subventionnés. Par qui? Au premier chef par la classe moyenne, en raison de son importance numérique. Mais la classe moyenne n'accepterait pas d'assumer sa part si les riches ne consentaient pas à participer de leur côté à la solidarité à un niveau plus élevé. Il résulte de ces raisons que l'impôt doit être progressif. D'un autre côté, il doit être modérément progressif. Sinon, un autre principe fondamental, le principe d'efficacité, serait violé. La classe riche aurait en effet la possibilité, au cas où l'impôt lui paraîtrait trop lourd, d'expatrier ses avoirs : un effet négatif du point de vue de la collectivité.

Sans doute certains citoyens s'opposent-ils à ce consensus et certains économistes recommandent-ils de revenir à un impôt proportionnel (flat tax), d'autres, peu nombreux, proposant purement et simplement d'abolir l'impôt sur le revenu. Mais c'est qu'ils s'en tiennent à des considérations de caractère instrumental et ignorent la dimension axiologique de la question : ils s'attachent aux effets et méconnaissent les principes.

Cette analyse en termes de raisons explique d'autres données, par exemple que les différences s'agissant du poids et de la progressivité de l'impôt sur le revenu entre les sociétés scandinaves et les sociétés de l'Europe continentale tendent à se réduire sous l'effet du principe d'efficacité.

56 Voir Stein Ringen, What Democracy Is for, Princeton, Princeton University Press, 2007. 


\section{Le changement de long terme comme effet de la rationalité ordinaire}

Durkheim $^{57}$ a insisté dans sa Division du travail social sur le fait que des tendances de long terme caractérisent les changements des sentiments moraux. Il tente de les saisir de façon synthétique par une distinction célèbre : ces tendances se traduiraient par une évolution dans la nature de la solidarité. Il la définit comme "mécanique " dans les sociétés traditionnelles et comme " organique " dans les sociétés modernes. Tout être humain a eu de tout temps et en tout lieu un sens de sa dignité et de ses intérêts vitaux et un sens critique lui permettant de juger des institutions sociales par rapport à ce critère. Mais l'intensification de la division du travail dans les sociétés modernes a entrainé que chacun y a rencontré au cours du temps des circonstances de plus en plus favorables à l'expression de sa demande de dignité et de respect pour ses intérêts vitaux, et a éprouvé un sens de plus en plus affirmé de son individualité. La division du travail a fait en d'autres termes que l'individualisme, que Durkheim voit comme un trait de la nature humaine, peut s'exprimer plus facilement dans les sociétés modernes. Cela explique qu'elles soient caractérisées par des tendances séculaires qu'il faut voir comme les effets d'un processus de sélection rationnelle des idées piloté par le politique sous le contrôle de l'opinion publique. Ainsi, le droit civil tend à s'étendre aux dépens du droit pénal, la sévérité des peines à s'atténuer, des catégories toujours plus nombreuses d'agissements à être dépénalisés et décriminalisés. La raison en est que, dès qu'une peine apparaît comme aussi efficace et plus légère, elle tend à être approuvée par le public. Ce schéma explique la tendance au rejet de la peine de mort qui se déploie dans les sociétés modernes.

Implicitement, les analyses profondes de La Division du travail social mettent en jeu la rationalité cognitive de l'individu quelconque. Ce que Durkheim, utilisant le vocabulaire qu'il avait à sa disposition, qualifie de "libre pensée " n'est en effet qu'un

57 Émile Durkheim, De la division du travail social, Paris, Presses universitaires de France, 1967 [1893]. 
autre nom pour désigner ce que les sciences sociales modernes appellent la rationalité cognitive. En faisant de la libre-pensée un trait de la nature humaine, il donne à l'opinion publique un rôle essentiel dans l'explication des phénomènes tendanciels qu'il met en évidence dans La Division du travail social.

\section{La théorie de la rationalité ordinaire permet de résoudre les apories de la théorie du choix rationnel}

Il résulte aussi de ce qui précède que l'un des avantages importants de la théorie de la rationalité ordinaire est qu'elle permet de résoudre bien des questions face auxquelles la théorie $d u$ choix rationnel est impuissante. On annexera ici quelques exemples aux précédents.

La théorie des jeux repose sur l'axiomatique de la théorie $d u$ choix rationnel. Or plusieurs situations d'interaction n'ont pas de solution dans le cadre de la théorie des jeux au sens où celle-ci est incapable de recommander aux acteurs une stratégie déterminée. Il s'agit notamment des structures perverses classiques comme le dilemme du prisonnier ou le jeu de l'assurance. Ces structures d'interaction ont inspiré une littérature surabondante. Or elles ne peuvent recevoir de solution aussi longtemps qu'on la recherche dans le cadre de la rationalité purement instrumentale postulée par la théorie du choix rationnel.

Les grands auteurs ont compris depuis longtemps que la solution de ces structures impose d'abandonner l'axiomatique de la théorie du choix rationnel.

Ainsi, Rousseau a bien vu que seule une innovation permet de sortir de l'ambiguïté créée par la structure désignée après lui jeu de l'assurance. La solution qu'il propose consiste à introduire un tiers personnage ayant le pouvoir de punir les acteurs qui trahiraient leur promesse de coopération.

Olson ${ }^{58}$ a bien vu qu'une action collective qui serait en principe utile à un groupe d'intérêt non organisé a des chances de ne pas

58 Mancur Olson, Logic of Collective Action, Cambridge (MA), Harvard University Press, 1965, traduction. française : La Logique de l'action collective, Paris, Presses universitaires de France, 1978 et Bruxelles, Éditions de l'Université de Bruxelles, 2011. 
avoir lieu, les acteurs étant alors pris au piège du dilemme $d u$ prisonnier. Il a montré que la solution de ce piège consiste, comme dans le cas des chasseurs de Rousseau, à imaginer des innovations permettant de détruire la structure perverse.

Le problème du carrefour a fini par prendre le statut d'un problème d'école dans la littérature inspirée par la théorie du choix rationnel: dois-je en l'absence supposée de règles laisser passer le véhicule se présentant en même temps que moi à un croisement sur l'autre voie? Ce problème n'a pas, lui non plus, de solution dans le cadre de l'axiomatique de la théorie du choix rationnel, puisque celle-ci ne permet pas aux automobilistes de trancher entre les deux équilibres de Nash engendrés par la structure. La solution consiste, ici encore, à introduire une innovation sous la forme de l'imposition d'une règle de priorité.

Et c'est une innovation, la menace de guerre des étoiles, qui a mis fin à la course aux armements qui s'était installée à l'issue de la seconde Guerre mondiale 59 .

La théorie de la rationalité ordinaire permet aussi d'expliquer les variations des sentiments moraux qu'observent les recherches quasi-expérimentales utilisant le jeu de l'ultimatum ou d'autres montages expérimentaux de même nature, comme le jeu $d u$ bien public. Dans ce dernier jeu, chacun choisit de s'attribuer une partie d'une somme et verse le reste au pot commun, sachant qu'en fin de partie, il recevra un certain pourcentage de la somme globale contenue dans le pot commun : dans tous les cas, des systèmes de raisons compréhensibles eu égard au contexte permettent de rendre compte des données observées. Dans le jeu de l'ultimatum, le sujet A fait une proposition sur le partage de $100 €$ entre lui-même et B. Si B l'accepte, le partage se fait selon la proposition de $\mathrm{A}$, sinon les $100 €$ restent dans la poche de l'expérimentateur. Dans la plupart des cas, A propose un partage 50/50. Il voit bien qu'il risquerait de ne rien avoir s'il tente de tirer la couverture à lui. Mais dans un contexte socio-culturel où

59 Raymond Boudon, Croire et savoir : penser le politique, le moral et le religieux, op. cit. 
la relation de concurrence est dominante, A est plus facilement tenté de profiter de la situation, comme dans la vie ${ }^{60}$.

\section{Aux racines de la sociologie analytique}

En un mot, on peut montrer que nombre de sociologues classiques et modernes ont suivi un paradigme défini par trois principes majeurs : singularisme méthodologique, individualisme méthodologique et équilibre cognitif. Il pose que les causes des actions et croyances individuelles résident dans les raisons que les acteurs sociaux ont de les adopter. Plus précisément, ceux-ci endossent une croyance représentationnelle ou normative dès lors qu'ils ont l'impression que cette croyance est fondée sur un système de raisons qui leur paraît meilleur que tout système alternatif. S'agissant des actions, elles comportent toujours des croyances.

Je ne puis développer comme il le mériterait un point essentiel, à savoir que nombre de croyances étant dépendantes du contexte, elles sont sources de concurrence voire de conflit, l'acteur social ayant facilement l'impression que ses croyances sont les bonnes. En même temps, les unes, étant objectivement supérieures aux autres sur certains sujets, tendent à s'imposer, sauf si des effets de contexte s'y opposent. On observe en effet que, sur maints sujets, les processus d'homogénéisation qui caractérisent les sociétés contemporaines se heurtent à des effets de contexte. L'homogénéisation n'exclut donc pas la diversité, pas plus que la recherche de l'universalité n'exclut la singularité. Le principe de la séparation des pouvoirs tend par exemple à être perçu comme de valeur universelle, mais sa mise en œuvre est différente d'une société démocratique à l'autre. La démocratie tend à être positivement valorisée, mais sa mise en œuvre se heurte à des effets de contexte dans bien des sociétés.

Étant donné sa force et son caractère d'évidence, le paradigme défini par les trois principes du singularisme méthodologique, de

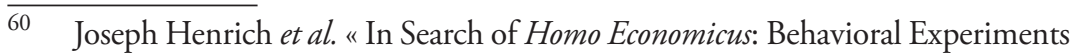
in 15 Small-scale Societies ", American Economic Review, vol. 91, n 2, 2001, p. $73-78$. 
l'individualisme méthodologique et de l'équilibre cognitif a été identifié sous des noms différents, on l'a dit : sociologie compréhensive (Weber), middle range theory (Merton) ou aujourd'hui erklärende Soziologie $\left(\mathrm{Esser}^{61}\right)$ et sociologie analytique (Hedström ${ }^{62}$, Manzo.). Pawson ${ }^{63}$ a montré de façon convaincante que le paradigme décrit par ces trois principes déchiffre la notion de middle range theory.

Ces remarques confirment, comme le suggère Helga Nowotny ${ }^{64}$, l'intérêt de la classification proposée par Michelle Lamont de quatre types fondamentaux de styles : compréhensif, constructiviste, positiviste et utilitaire. Bien sûr, d'autres définitions de ces styles cognitifs peuvent être proposés. Et il faut reconnaître le caractère changeant des préférences pour tel ou tel style. J'ajouterai seulement que si le style compréhensif est dominant, cela n'est pas sans raisons. J'irai même plus loin. Le paradigme à trois principes définit un type particulier de sociologie, le type qui peut être dit scientifique au sens où il propose d'expliquer tout phénomène social plus ou moins énigmatique comme l'effet d'un ensemble articulé de données factuelles et de lois avérées. Or expliquer un phénomène énigmatique en en faisant le résultat de lois avérées et de données de fait est exactement ce que font tous les physiciens, biologistes ou astronomes.

Sans doute tous les sociologues n'épousent-ils pas ce paradigme. J'ai évoqué le contraste entre la conception implicite de la sociologie que se font Weber, Durkheim et leurs continuateurs et la conception holiste qu'illustrent Le Bon ou aujourd'hui Ulrich Beck ou Zygmunt Bauman. À côté de ces deux types de sociologie, bien d'autres pourraient être identifiés et l'on peut

61 Voir Paul Hill et al., Hartmut Esser, Erklärende Soziologie. Kintroversen und Perspektiven, Auflage, Campus Verlag, 2009.

62 Peter Hedström, Dissecting the Social: On the Principles of Analytical Sociology, Cambridge, Cambridge University Press, 2005.

63 Ray Pawson, "On the Shoulders of Merton. Boudon as the Modern Guardian of Middle-Range Theory ", dans Mohamed Cherkaoui et Peter Hamilton (dir.), Raymond Boudon: A life in Sociology. Essays in Honour of Raymond Boudon, 4 volumes, Oxford, Bardwell, 2009, IV, p. 317-334.

64 Helga Nowotny, "La Sociologie a perdu son assise politique au profit de l'économie ", Commentaire, hiver 2011-2012, p. 1053-1054. 
proposer maintes classifications différentes de celle de Michelle Lamont. J'ai moi-même distingué dans mon discours inaugural à l'Académie européenne de sociologie ${ }^{65}$ la sociologie explicative, la sociologie caméraliste et la sociologie critique. Mais il existe aussi une sociologie descriptive, souvent fascinante, qui explique que la sociologie paraisse dans ce cas plus proche de l'art que de la science, comme le suggèrent Walter Garrison Runciman ${ }^{66}$ et Brigitte Nedelmann ${ }^{67}$. Il y a aussi une sociologie théorique : celle qui adopte un point de vue réflexif sur la sociologie existante. La Structure of Social Action de Parsons ou la théorie empirique de l'action de Lazarsfeld en sont des illustrations canoniques. Il y a aussi une sociologie critique qui tend à rechercher des théories utiles à la défense de telle ou telle cause. Il y a une sociologie davantage imprégnée de scientisme que d'esprit scientifique. Ce type - le style positiviste selon Lamont - est illustré par la sociologie structurale, la mémétique, la sociobiologie ou la sociologie qui met tous ses espoirs dans le développement des neurosciences ${ }^{68}$. Ces variantes ont en commun de supposer que le comportement humain est l'effet de causes matérielles, à savoir de causes sociales, psychologiques ou biologiques qui, à l'instar de celles qui président aux mécanismes de la digestion, échapperaient totalement, non seulement au contrôle de l'esprit humain, mais à la conscience. Comme Erner l'a brillamment démontré, la sociologie française des années 1970-1990 doit son succès à ce qu'elle a suscité une réaction compassionnelle en décrivant les comportements auto-destructeurs d'échec scolaire ou de délin-

65 Raymond Boudon, La Sociologie comme science, op. cit.; Boudon, Raymond, (2011b), "Les sciences humaines peuvent-elles échapper au naturalisme? ", dans Bronner, Gérald et Romy Sauvayre (dir.), Le naturalisme dans les sciences sociales, Paris, Hermann, 2011, p. 23-50.

66 Runciman, Walter Garrison, "Précisions ", Commentaire, n 136, hiver 2011-2012, p. 1073.

67 Nedelmann, Birgitta, "Briser la base nationale de la profession ", Commentaire, $\mathrm{n}^{\circ} 136$, hiver 2011-2012 p. 1049-1052.

68 Boudon, Raymond, "Les Sciences humaines peuvent-elles échapper au naturalisme ", dans Gérald Bronner et Romy Sauvayre (dir.), Le Naturalisme dans les sciences sociales, Paris, Hermann, p. 23-50; Raymond Boudon, Croire et savoir : penser le politique, le moral et le religieux, op. cit. 
quance comme un effet de la « domination " sociale ${ }^{69}$. Finalement, on discerne une catégorie résiduelle qu'on peut qualifier de words-words-words sociology. Au total, la sociologie contemporaine est hautement diverse. Malheureusement, cela conduit de nombreux manuels à se présenter comme des catalogues commerciaux, juxtaposant les produits les plus divers. Il me semble que l'intérêt pour le mouvement dit de la sociologie analytique est le symptôme du sentiment croissant d'inconfort induit par cette situation et de la croyance selon laquelle la sociologie peut obéir à l'esprit scientifique comme toutes les autres sciences.

J'ai cependant l'impression que les manuels présentant la sociologie analytique ont le défaut de cacher les principes sous des détails techniques d'importance secondaire et qu'ils manquent d'identifier clairement le paradigme commun aux explications de phénomènes sociaux qui se sont irréversiblement imposées, à savoir le paradigme défini par les trois principes du singularisme méthodologique, de l'individualisme méthodologique et de l'équilibre cognitif.

Munie de ses trois principes, la sociologie analytique permet d'éliminer les vues simplistes qui voient les sociétés contemporaines comme vouées soit à l'homogénéisation soit au conflit culturel endémique, car des effets de contexte variés limitent et diversifient l'action des facteurs favorisant l'homogénéisation.

$\overline{69}$ Guillaume Erner, La Société des victimes, Paris, La Découverte, 2006. 


\section{Références}

Bauman, Zygmunt, Does Ethics Have a Chance in a Word of Consumers?, Cambridge (MA), Harvard University Press, 2008.

Beattie, John, Other Cultures, Londres, Cohen \& West, 1964.

Bechtel, Guy, La Sorcière et l'Occident, Paris, Plon, 1997.

Beck, Ulrich, Risk Society: Towards a New Modernity, London, Sage, 1992.

Borlandi, Massimo, "Durkheim et la psychologie ", dans Raymond Boudon (dir.), Durkheim fut-il durkheimien?, Actes du colloque organisé les 4 et 5 novembre 2008 par l'Académie des sciences morales et politiques, Paris, Colin, coll. "Recherches », 2011, p. 55-80.

Boudon, Raymond, Croire et savoir : penser le politique, le moral et le religieux, Paris, Presses universitaires de France, 2012.

Boudon, Raymond, La Sociologie comme science, Paris, La découverte, coll. "Repères », 2010.

Boudon, Raymond, "Les Sciences de la nature vues par les sciences sociales ", communication au colloque international et multidisciplinaire de l'Académie européenne interdisciplinaire des sciences sur Théories et modèles en sciences sociales, 28-29 novembre 2011, à paraitre en 2012.

Boudon, Raymond, "Les Sciences humaines peuvent-elles échapper au naturalisme ", dans Gérald Bronner et Romy Sauvayre (dir.), Le Naturalisme dans les sciences sociales, Paris, Hermann, 2011, p. 23-50.

Boudon, Raymond, Tocqueville aujourd'hui, Paris, Odile Jacob, 2005.

Cherkaoui, Mohamed, "La Complexité du social et la méthode sociologique selon Durkheim ", dans Boudon Raymond (dir.), Durkheim fut-il durkheimien?, Actes du colloque organisé les 4 et 5 novembre 2008 par l'Académie des sciences morales et politiques, Paris, Colin, coll. « Recherches », 2011, p. 81-98.

Cherkaoui, Mohamed et Peter Hamilton (dir.), Raymond Boudon : A life in sociology. Essays in honour of Raymond Boudon, 4 volumes, Oxford, Bardwell, 2009.

Coleman, James, Foundations of Social Theory, Cambridge (MA), Harvard University Press, 1990.

Coleman, James, Elihu Katz et Herbert Menzel, Medical Innovation, Indianapolis, Bobbs-Merrill, 1966.

Crapez, Marc, Défense du bons sens, Paris, Éditions du Rocher, 2004.

Demeulenaere, Pierre, (dir.), Analytical Sociology and Social Mechanisms, Cambridge, Cambridge University Press, 2010. 
Demeulenaere Pierre, "Mécanismes, causalité, régularités de comportements et normes sociales ", dans Gérald Bronner et Romy Sauvayre (dir.), Le Naturalisme dans les sciences sociales, Paris, Hermann, 2011, p. 167-182.

Durkheim, Émile, De la division du travail social, Paris, Presses universitaires de France, 1967 [1893].

Durkheim, Émile, Les Formes élémentaires de la vie religieuse, Paris, Presses universitaires de France, 1979 [1912].

Erner, Guillaume, La Société des victimes, Paris, La Découverte, 2006.

Henrich, Joseph et al "In Search of Homo Economicus: Behavioral Experiments in 15 Small-Scale Societies ", American Economic Review, vol. $91, \mathrm{n}^{\circ}$ 2, 2001, p. 73-78.

Hedström, Peter, Dissecting the Social: On the Principles of Analytical Sociology, Cambridge, Cambridge University Press, 2005.

Hill, Paul et al, Hartmut Esser, Erklärende Soziologie. Kintroversen und Perspektiven, Auflage, Campus Verlag, 2009.

Horton, Robin, Patterns of Thought in Africa and the West, Cambridge, Cambridge University Press, 1993.

Hubert, Henri et Marcel Mauss, "Esquisse d'une théorie générale de la magie ", L'Année sociologique, $\mathrm{n}^{\circ}$ 7, 1902-1903, p. 1-146. Repris dans Marcel Mauss, Sociologie et anthropologie, précédé d'une «Introduction à l'œuvre de Marcel Mauss » par Claude Lévi-Strauss, Paris, Presses Universitaires de France, 1950, p. 1-141.

Inglehart, Ronald et al, Human Values and Beliefs: A Cross-Cultural Sourcebook: Political, Religious, Sexual, and Economic Norms in 43 Societies: Findings from the 1990-1993 World Values Survey, Ann Arbor, The University of Michigan Press, 1998.

Katz, Elihu et Paul Lazarsfeld, Personal Influence, Glencoe (Ill), The Free Press 1955 et Londres, Transaction, 2006.

Kuhn, Thomas S., The Structure of Scientific Revolutions, Chicago, University Chicago Press, 1962.

Lakatos, Imre, «Falsification and the Methodology of Scientific Research Programs ", dans Imre Lakatos, et Alan Musgrave (dir.), Criticism and the Growth of Knowledge, Londres, Cambridge University Press, 1965, p. 91-195.

Le Bon, Gustave, Psychologie des foules, Paris, Alcan, 1895.

Lepenies, Wolf, Die drei Kulturen, Munich, Hanser, 1985. En français : Les trois cultures, Paris, Maison des sciences de l'homme, 1991.

Lévy-Bruhl, Lucien, La Mentalité primitive, Paris, Presses universitaires de France, 1960 [1922]. 
Luckmann, Thomas, The Invisible Religion: The Problem of Religion in Modern Society. New York, Macmillan, 1967.

Manzo, Gianluca, «Analytical Sociology and Its Critic », European Journal of Sociology, vol. 51, n 1, 2010, p.129-170.

Manzo, Gianluca, La Spirale des inégalités. Choix scolaires en France et en Italie au XXe siècle, Paris, Presses de l'Université de Paris-Sorbonne, 2009.

Mead, George Herbert, L'Esprit, le soi, la société, Paris, Presses universitaires de France, 2006 [1934].

Mises, Ludwig von, Human Action, New Haven (CT), Yale University Press, 1949. En français : L'Action humaine, Paris, Presses Universitaires de France, 1985.

Nedelmann, Birgitta, "Briser la base nationale de la profession ", Commentaire, $\mathrm{n}^{\circ}$ 136, hiver 2011-2012 p. 1049-1052.

Needham, Rodney, Belief, Language and Experience, Oxford, Blackwell, 1972.

Nowotny, Helga, «La Sociologie a perdu son assise politique au profit de l'économie ", Commentaire, hiver 2011-2012, p. 1053-1054.

Olson, Mancur, Logic of Collective Action, Cambridge (MA), Harvard University Press, 1965, traduction française : La Logique de l'action collective, Paris, Presses universitaires de France, 1978 et Bruxelles, Éditions de l'Université de Bruxelles, 2011.

Parsons, Talcott, The Structure of Social Action: A Study in Social Theory with Special Reference to a Group of Recent European Writers, New York, McGraw-Hill, 1937. Nouvelle édition, Glencoe (Ill), The Free Press, 1949.

Parsons, Talcott et al., Theories of society. Foundations of modern sociological theory, Glencoe, Ill., The Free Press, 2 volumes, 1961.

Pawson, Ray "On the Shoulders of Merton. Boudon as the Modern Guardian of Middle-Range Theory ", dans Mohamed Cherkaoui et Peter Hamilton (dir.), Raymond Boudon: A life in Sociology. Essays in Honour of Raymond Boudon, 4 volumes, Oxford, Bardwell, 2009.

IV, p. 317-334.

Popper, Karl, "The Myth of the Framework ", dans Freeman, R. Edward (dir.), The Abdication of Philosophy. Philosophy and the Public Good, La Salle (Ill), Open Court, 1976, p. 23-48.

Rawls, John, A Theory of Justice, Cambridge (MA), Harvard University Press, 1971. 
Reynaud, Jean-Daniel, "Pierre Bourdieu (1930-2002) : restituer aux hommes le sens de leurs actes ", Revue française de sociologie, vol. 43, $\mathrm{n}^{\circ} 1$, p. I-IV, 2002.

Ringen, Stein, What Democracy Is for, Princeton, Princeton University Press, 2007.

Runciman, Walter Garrison, "Précisions ", Commentaire, n 136, hiver 2011-2012, p. 1073.

Sahlins, Marshall, Culture and Practical Reason, Chicago, University of Chicago Press, 1976.

Simmel, Georg, Die Probleme der Geschichtsphilosophie,Munich, Mohr, 1892.

Smith, Adam, An Inquiry into the Nature and Causes of the Wealth of Nations, $7^{\mathrm{e}}$ édition, Londres, Strahan and Cadell, 1976 [1793].

Tåhlin, Michael, "Laissez-moi ma liberté. Pour une sociologie pluraliste ", Commentaire, hiver 2011-2012, p. 1077-1082.

Thomas, Keith, Religion and the Decline of Magic: Studies in Popular Beliefs in Sixteenth and Seventeenth Century England, London, Penguin Books, 1973. Harmondsworth, 1973. Tocqueville, Alexis de, La démocratie en Amérique II, in Tocqueville, Paris, R. Laffont, collection Bouquins, 1986 [1840].

Tocqueville, Alexis de, La Démocratie en Amérique II, Paris, Gallimard, 1986 [1840].

Walzer, Michael, Sphères de justice, Une défense du pluralisme et de l'égalité, Paris, Seuil, 1997.

Weber, Max, Économie et société, Paris, Plon, 1971 [1922].

Weber, Max, Gesammelte Aufs̈̈tze zur Religionssoziologie, Munich, Mohr, 1986 [1920].

Weber, Max, Gesammelte Aufsätze zur Wissenschaftslehre, Tübingen, Mohr, 1922. En français, Essais sur la théorie de la science, Paris, Plon, 1965.

Wiggershaus, Rolf, The Frankfurt School, Norristown (PA), 1995. En français : L'École de Francfort, Paris, Presses universitaires de France, 1993, présentation et traduction de Lilyane Deroche-Gurcel.

Wittgenstein, Ludwig, "Bemerkungen über Frazer's, The Golden Bough », Synthese, no 17, 1967, p. 233-253. 